\title{
Contemporary Approach to Determination of Magnetic Induction in Wind Generator Air Gap
}

\author{
A. Elez ${ }^{1}$, B. Tomičić ${ }^{2}$ and B. Takač ${ }^{1}$ \\ ${ }^{1}$ Končar-Institut za elektrotehniku d.d. \\ Fallerovo šetalište 22, 10000 Zagreb (Croatia) \\ Phone/Fax number:+00385 1365 6290, e-mail: elez@ koncar-institut.hr, btakac@ koncar-institut.hr \\ ${ }^{2}$ Končar-Generatori i motori d.d. \\ Fallerovo šetalište 22, 10000 Zagreb (Croatia) \\ Phone/Fax number:+00385 1365 5135, e-mail: btomicic@koncar-gim.hr
}

\begin{abstract}
Contents. The paper describes a system for measuring of magnetic induction on the wind generating unit. The manner of operation and the application of the Hall effect probe are detailed. Dynamic electromagnetic field computing results are shown through application of MKE on the synchronous generator model installed in a windmill unit and compared to the actual metering results. Two subsequent magnetic field calculations on the modified model were made to be used for analyses of the obtained results. Influences of air gap forms and saturation to time dependence of magnetic induction at a point within the machine are explained.
\end{abstract}

Key words. Hall effect probe, synchronous generator, magnetic induction, MKE.

\section{Introduction}

Awareness of rotating machine electromagnetic phenomena is of an utmost importance for their optimal designing and defining of permissible load limits under individual operating conditions.

For designing of rotating machines powerful analytic tools are used nowadays that, using MKE with hypothetic initial and marginal conditions, enable calculation of electromagnetic fields in realistic machine structures. Simulation under all operation regimes are also possible including the faulty ones as well. In this matter, the correctness of each calculation depends on suitability of the model itself as well as the suitability of selected initial and marginal conditions to the real facility that is under the process of designing. In addition to the stated, the designer has also to keep in mind that certain models and input hypothesis are valid within a certain scope of structure measures of the real facility.

Therefore the following tasks emerge to be a must:

- determination of preciseness of electromagnetic phenomena calculations in comparison with the metering results

- determination of the scope of varying of input data within which the calculation is still within the sufficient preciseness compared to metering results.

Within the frame of resolving the first item, i.e. determination of the preciseness of electromagnetic phenomena calculations comprehensive metering were performed and reported in this paper.
Metering was performed by application of techniques and technical solutions incorporated in rotating machines monitoring systems. Rotating machines monitoring has been generally applied and installed to monitor predefined machine parameters, and to apply preventive activities as necessary, and in such a way to achieve a significant increase of safety and security of people and reliability of the plant. Monitoring system application also decreases plant maintenance costs and prolong its complete lifetime cycle.

One of the possible methods for machine condition monitoring is measuring of magnetic fields. It is often used in combination with other measuring methods. Probes are usually installed at points of interest, and measuring signals are processed in measuring processing units. Depending on the level of complexity of the used measuring and data processing units, the needed quality of measuring may be achieved.

A developed measuring system, as well as performed measuring has enabled comparison of results achieved by calculations and by measuring.

\section{Measuring and Data Processing System}

Fig 1. shows the measuring and data processing system of the wind generating unit KO-VA/57/1. The system consists of a measured facility, measuring probe, insulation attenuators, a unit for receipt and processing of data and a central computer.

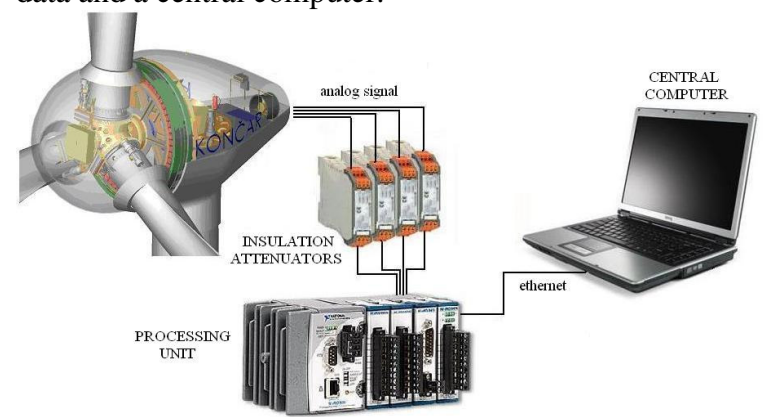

Fig. 1. Electromagnetic induction measuring system applied to wind generator 
The measured facility is a synchronous three phase generator with variable rotation speed having the following nominal data:

Power: $1000 \mathrm{kVA}$,

Line voltage: $690 \mathrm{~V}$,

Current: 867 A,

Power factor: 0,95 ,

Frequency: 14,5 Hz,

Number of poles: 60 ,

Rotation velocity: $29 \mathrm{rpm}$.

Hall effect probes have been installed to measure magnetic induction in the active part of the machine. The electric connection of the Hall effect probe is shown on the Figure 2.

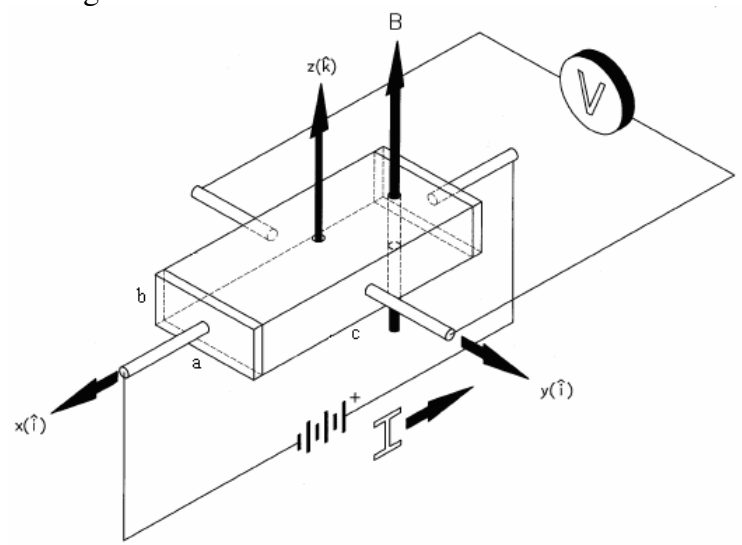

Figure 2. Hall effect probe electric connection

When the Hall effect probe is connected to an outside voltage source, the control current will run thought it. If the Hall effect probe is placed in a magnetic field at the same time, a power will influence electric charges within the probe material generating a potential difference at diagonal terminals.

Pursuant the expert literature [1], the potential difference or Hall Voltage is calculated in accordance with the expression:

$$
V_{H}=\frac{I \cdot B}{N \cdot q \cdot b}
$$

where:

I - is the current running through the probe,

$\mathrm{B}$ - is a magnetic induction component normal to the measuring surface,

$\mathrm{N}-$ is the number of electric charges,

$\mathrm{q}$ - is a free charge (electrons or cavities depending on the type of material used for the Hall effect probe),

$b-$ is the height of the Hall effect probes.

The Hall effect coefficient that depends completely on the type of material making the Hall effect element is calculated on the bases of the following formula:

$$
R_{H}=\frac{1}{N \cdot q}
$$

On the basis of the equation (1), the expression for magnetic induction, i.e. the magnetic flux density is derived:

$$
B=-\left(\frac{b}{R_{H}}\right) \frac{V_{H}}{I}
$$

The equation (3) gives only the magnetic induction component that is vertical to the Hall effect probe surface.

Hall effect probes measure magnetic induction per unit of the surface. The contemporary technology has enabled the dimensions of the probes themselves to be extremely small (Figure 3a.).

Hall effect probes have a wide scope of application in different areas. They are not sensible to the majority of distortions such as for example humidity, vibrations, air pollution and lightening and therefore they are completely capable for this application. Figure $3 b$. shows a probe prepared for installation.

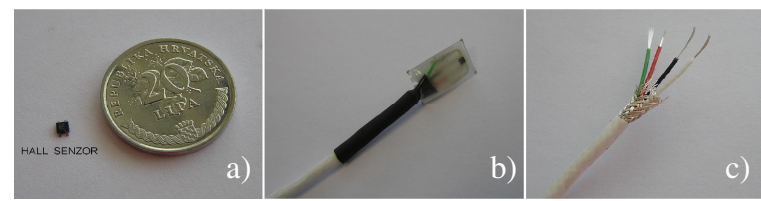

Figure 3. a) Hall effect probe

b) Hall effect probe prepared for installation

c) signal cable

Transmission of signals from the Hall effect probe to the measuring unit is performed by a signal cable. The signal cable (Figure 3c.) consists of interwoven conductors having telephone insulation and being resistant to high temperature. In order to eliminate possible disturbances that may be inducted in conductors the used cable has to be screened.

On the market, Hall effect probes are available at a very low price. They are not accompanied with measuring lists. Therefore calibration has to be performed for each probe and their output characteristics determined as well. The Hall effect probe is a linear element meaning that the output voltage is proportionate to the measured magnetic induction and the control current. Output characteristic is different for each probe. The Rotational Machines Laboratory of the Končar - Electric Engineering Institute Inc. has realised calibration of Hall effect probes for our needs, and the typical values of sensibility are within the scope of $1 \mathrm{~V} / \mathrm{T} \pm 5 \%$ at the control current of $5 \mathrm{~mA}$.

If the distance between probes and the data collecting and processing unit is relatively large, it is desireable to amplify the signal at the point that is the closest to its installation using a standard isolation amplifier.

Data obtained from the probes, i.e. isolation amplifiers are transmitted through signal cables to the data collecting and processing unit. That unit shall be selected in accordance with the measuring requirements. Measuring may be of a permanent type with a protection function, or of an intermittent type with a diagnostic purpose. The used data collecting and processing unit is a small, reliable and independent unit with FPGA chip enabling uncomplicated hardware programming from a graphic programming language. The measuring unit comprises a $400 \mathrm{MHz}$ processor that performs any software application in a predetermined manner. The unit is of a modular type meaning that the user itself 
makes the hardware configuration using various analogous and digital input and output modules. Processed and digitalised data are being sent to the central computer via Ethernet.

The central computer is used for control and management of data collecting unit settings such as data processing selection, hardware settings and communication settings. In addition it is used to generate displays showing the real time and previously memorised data. The access to the central computer data is enabled by the help of a client application either locally or remotely.

\section{Magnetic Induction Calculation based on Finite Element Method}

Applying software packages that implement MKE for magnetic field calculations, we will calculate magnetic induction wave forms at points where Hall probes are installed. The results will be compared to the measured values later on.

For MKE application calculations it is necessary to introduce the following presumptions:

- Machine windings are symmetrically arranged around every pole; they have the same number of conductors and the same resistance enabling the analysis of only one pole,

- The relationship between the length and the diameter of the generator enables the application of a two dimension model,

- The influence of the skin effect within the armature and the excitation winding is neglected,

- Iron electric conductivity is neglected and as a consequence the influence of eddy currents and interbar rotor currents are excluded,

- Features of the installed material are isotropic, hysterisis curve is neglected, while the permeability of iron depends on the magnetic field strength,

- Rotation velocity of the rotor that changes during transient conditions is neglected due to a large inertia mass,

- All machine windings are connected to ideal sources.

Figure 4a. shows the model of a generator pole for a windmill farm made by the software package MagNet [4]. The model consists of a static and a rotating part.

The static part consists of a stator core made of dynamo laminations with slots in which three phase winding copper conductors are placed. The number of coils per pole and per phase amounts to 2, and they are made without cutting. The stator core is placed in a housing made of steel, while an air duct is made between the housing and the stator core.

The rotating part is made of a pole wheel with poles made of dynamo laminations. An excitation winding coil is wound up around each pole. The pole shoe comprises 6 holes in which the damping winding made of round copper bars is inserted. The pole is placed on a steel hub. A dynamic model, which also takes into consideration rotation of the rotor, has been applied for calculation of magnetic field in the generator, while the calculation of the field has been founded on expressions taken form the technical literature [4]:

$$
\begin{aligned}
& \oint_{C} E \cdot d l=-\int_{S} \frac{\partial B}{\partial t} \cdot n \cdot d S=-\frac{d}{d t} \int_{S} B \cdot n \cdot d S \\
& \oint_{C} H \cdot d l=\int_{S} J \cdot n \cdot d S \\
& \oint_{S} B \cdot n \cdot d S=0
\end{aligned}
$$

where:

E - is electric field strength in conductors,

$\mathrm{B}$ - is magnetic induction,

$\mathrm{H}-$ is electric field strength,

$\mathrm{J}-$ is current density,

$\mathrm{C}-$ is curve defining surface $\mathrm{S}$,

$\mathrm{S}$ - is surface within which the filed is calculated, $\mathrm{n}-$ is unit vector normal to the surface $\mathrm{S}$.

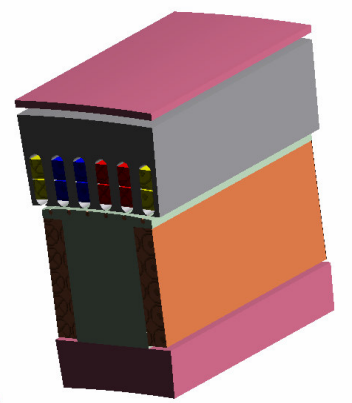

a)
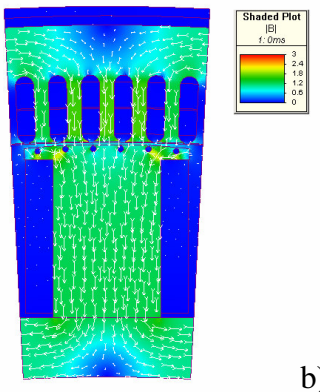

b)

Fig. 4.a) Model of the windmill unit generator, b) Magnetic field at nominal voltage

The calculation is made during the time period corresponding to one period of output voltage for different values of excitation current. The velocity of rotation is constant all the time and has the nominal value. The result of the magnetic field calculation in idling with excitation current at which the nominal value voltage is induced in the stator winding as shown on Figure 4b.

\section{Measuring}

Hall probes have been installed at the stator core tooth (Fig. 5a), and at the rotor pole shoe (Fig. 5b). Data from the stator probes are directly connected to the measuring unit, while data from the rotor probes are transmitted in a wireless manner to the stator in the central computer.
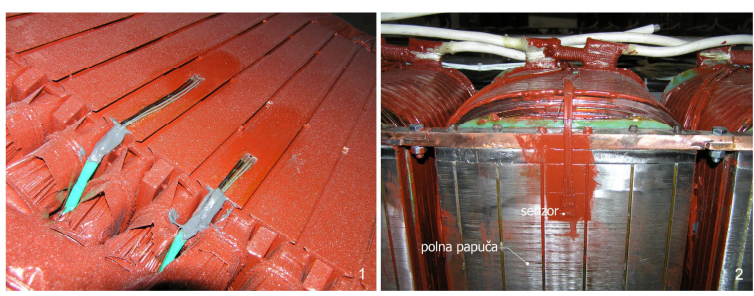

Fig. 5. a) Probes for measuring of magnetic induction in the air gap installed at the stator core tooth,

b) a probe for measuring of magnetic induction in the air gap installed at the rotor pole shoe

Magnetic induction time dependence at the top of the stator core tooth, for idling at the excitation current of 53 A, is shown on Figure 6 . The frequency causing emission of signals amounts to $5 \mathrm{kHz}$. At the generator rotation 
frequency amounting to $14 \mathrm{~Hz}, 357$ dots are generated for one signal half period that is sufficient for a high quality description of the signal.

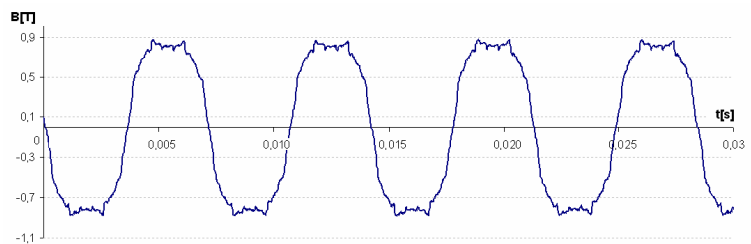

Figure 6. Display of magnetic induction time dependency at the top of the tooth obtained by measuring during idling at the current of $53 \mathrm{~A}$

Figure 7. shows time record of magnetic induction obtained by measuring on the top of the stator core tooth (curve 2), and on the top of the pole shoe (curve 1), during idling at the excitation current of $53 \mathrm{~A}$.

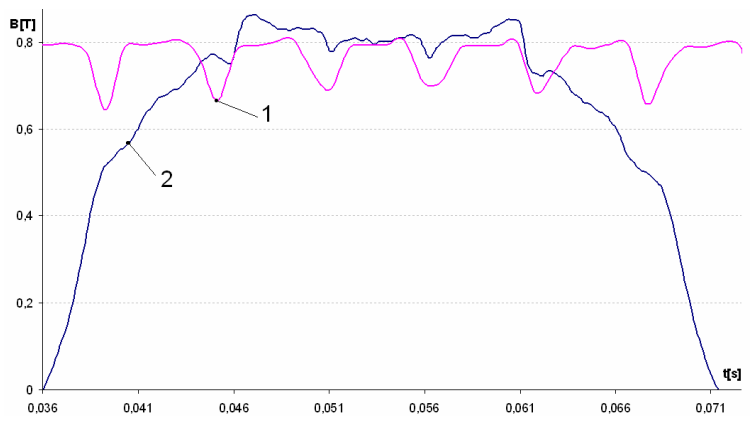

Figure 7. Magnetic induction arrangement over the pole pitch obtained by measuring during idling at the excitation current of $53 \mathrm{~A}$ :

Curve 1- on the top of the pole shoe,

Curve $2-$ on the top of the stator core tooth

\section{Comparison of Calculation Results to Measuring Results}

Fig. 8. shows calculated and measured values of time dependence of magnetic induction on the top of the stator core tooth.

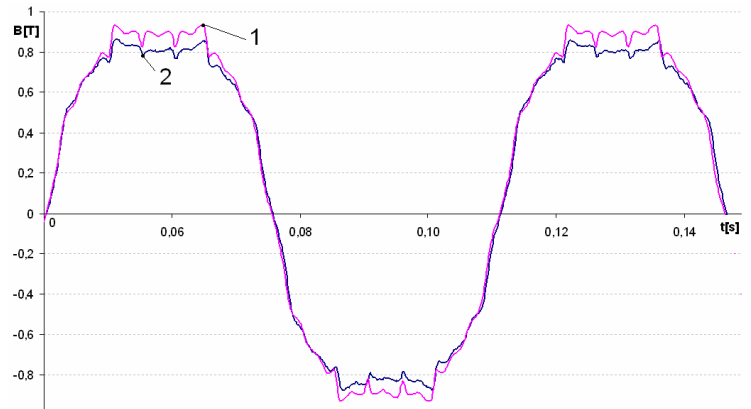

Fig. 8. Display of time dependence of magnetic induction on the top of the stator core tooth during idling at the excitation current of $53 \mathrm{~A}$ :

Curve 1 - finite element method calculation,

Curve 2 - measurements by Hall probe

Looking at the same figure we can see that magnetic induction time dependences obtained by a calculation and by measurement are similar regarding their form, but that there are some differences in their momentary values. The maximum value of magnetic induction in the air gap obtained by the calculation during idling at excitation current of $53 \mathrm{~A}$ amounts to $0,93 \mathrm{~T}$, while the maximum measured value of magnetic induction is $0,89 \mathrm{~T}$ at the same value of excitation current. It can be derived from the stated that the maximum values of magnetic inductions obtained by the calculation and by measuring at the excitation current of $53 \mathrm{~A}$ differ by $4,5 \%$.

Besides, deviations of magnetic induction maximum values at lower values of the excitation currents have been also analysed. Figure 9. shows time dependence of magnetic induction at the top of the tooth during idling for excitation currents of $25 \mathrm{~A}$ and $53 \mathrm{~A}$.

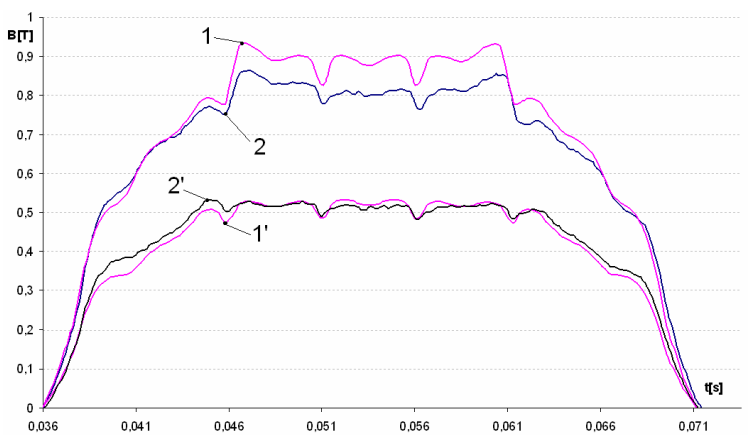

Figure 9. Display of time dependence of magnetic induction on the top of the stator core tooth during idling:

Curve 1- MKE calculation for excitation current $53 \mathrm{~A}$,

Curve 2-Hall probe measuring at excitation current 53A,

Curve 1'- MKE calculation for excitation current $25 \mathrm{~A}$,

Curve 2'- Hall probe measuring at excitation current 25A,

The maximum value of magnetic induction in the air gap obtained by calculation during idling at excitation current of $25 \mathrm{~A}$ amounts to $0,526 \mathrm{~T}$, while at the same excitation current the maximum measured value of magnetic induction amounts to $0,527 \mathrm{~T}$, making a deviation of $0,1 \%$.

Fig. 10. shows calculated and measured values of magnetic induction time dependence on the top of the rotor pole shoe.

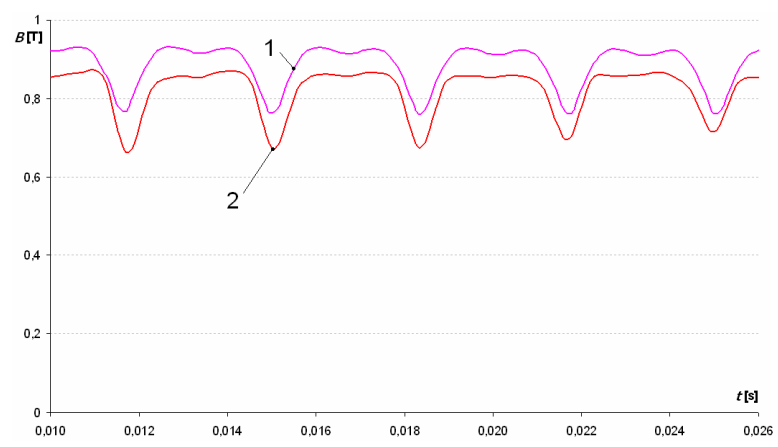

Figure 10. Display of magnetic induction time dependence on the top of rotor pole shoe during idling at nominal voltage: 1-MKE calculation,

2-measuring by Hall probe 
Figure 11. shows magnetic induction time dependence on the top of the pole shoe during idling at excitation current of 53 $\mathrm{A}$ and $25 \mathrm{~A}$.

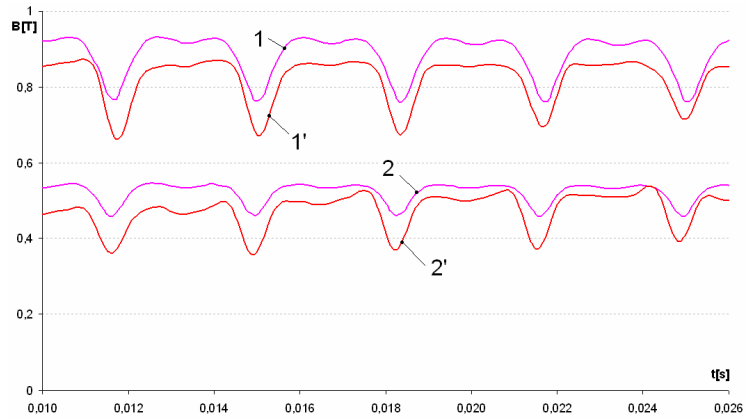

Figure 11. Display of magnetic induction time dependence on the top of rotor pole shoe during idling:

Curve 1- MKE calculation for excitation current $53 \mathrm{~A}$,

Curve a 1'- measured by Hall probe at excitation current 53

A,

Curve 2- MKE calculation for excitation current $25 \mathrm{~A}$,

Curve 2'- measured by Hall probe at excitation current $25 \mathrm{~A}$.

On the same figure, it can be seen that magnetic induction time dependences on the top of the rotor pole shoe obtained by calculation and by measuring correspond completely in form. Nevertheless, in the same manner as in the previous comparison, there are differences between maximum values. The maximum value of magnetic induction in the air gap obtained by calculation during idling at excitation current of $53 \mathrm{~A}$ amounts to $0,91 \mathrm{~T}$, while at the same value of excitation current, the maximum measured value of magnetic induction is $0,87 \mathrm{~T}$, making a deviation of $4,6 \%$.

Table 1 provides for a presentation of maximum values of magnetic inductions obtained by calculation and by measuring for different excitation currents, as well as deviations form calculations and measuring. Deviations are calculated on the basis of the following formula:

$$
\left(\frac{B_{P m}}{B_{M m}}-1\right) \cdot 100
$$

where:

$\mathrm{B}_{\mathrm{Pm}}$ - is maximum value of magnetic induction obtained by calculation,

$\mathrm{B}_{\mathrm{Mm}}-$ - is maximum value of magnetic induction obtained by measuring.

Table 1. Display of maximum values of magnetic induction obtained by calculation and measuring for various excitation currents and deviations from calculations and measuring

\begin{tabular}{|c|c|c|c|c|c|c|c|c|}
\hline \multirow{3}{*}{ No. } & \multirow{2}{*}{\multicolumn{2}{|c|}{ Excitation current }} & \multicolumn{4}{|c|}{ Maximum values of magnetic induction [T] } & \multirow{2}{*}{\multicolumn{2}{|c|}{ Deviation [\%] }} \\
\hline & & & \multicolumn{2}{|c|}{ Stator } & \multicolumn{2}{|c|}{ Rotor } & & \\
\hline & {$[\mathrm{A}]$} & [\%] & Calculation & Measuring & Calculation & Measuring & Rotor & Stator \\
\hline 1 & 3,5 & 6,6 & 0,073 & 0,069 & 0,073 & 0,077 & 5,7 & $-5,19$ \\
\hline 2 & 10,7 & 20,19 & 0,225 & 0,22 & 0,221 & 0.229 & 2,2 & $-3,94$ \\
\hline 3 & 14,6 & 27,55 & 0,317 & 0,31 & 0,302 & 0,305 & 2,2 & $-0,98$ \\
\hline 4 & 20 & 37.74 & 0.427 & 0.42 & 0.413 & 0.412 & 1.6 & 0.24 \\
\hline 5 & 25,5 & 48,11 & 0,535 & 0,532 & 0,526 & 0,523 & 0.5 & 0,57 \\
\hline 6 & 31 & 58,49 & 0.65 & 0,648 & 0,634 & 0,623 & 0,3 & 1,77 \\
\hline 7 & 36,4 & 68,6 & 0,74 & 0,72 & 0,726 & 0.712 & 2.4 & 1,97 \\
\hline 8 & 43 & 81,13 & 0,809 & 0,783 & 0,818 & 0,798 & 3,2 & 2,51 \\
\hline 9 & 48,5 & 91,51 & 0,872 & 0,837 & 0,883 & 0.849 & 4 & 3.8 \\
\hline 10 & 53,3 & 100,47 & 0,917 & 0,872 & 0,932 & 0,897 & 5,1 & 3,9 \\
\hline 11 & 58,56 & 110,49 & 0,945 & 0,895 & 0.984 & 0,941 & 5.2 & 4,53 \\
\hline 12 & 62,74 & 118,38 & 0,968 & 0,911 & 1.016 & 0,964 & 5,8 & 5,39 \\
\hline 13 & 67.54 & 127,43 & 1.016 & 0.949 & 1.056 & 0.991 & 6.9 & 6.56 \\
\hline
\end{tabular}

Figure 12. shows a deviation curve of maximum values of magnetic induction obtained by calculation and measuring for excitation current from 0 to $125 \%$ of the nominal.

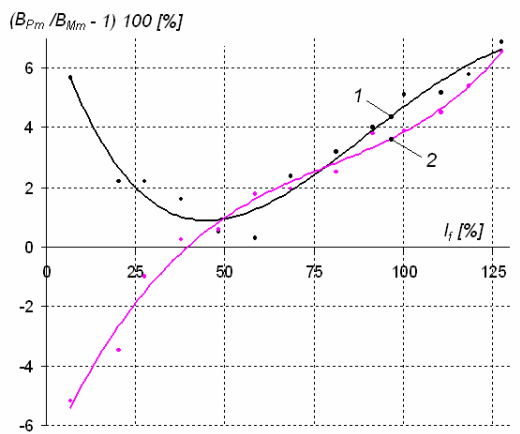

Figure 12. Deviations from maximum values of magnetic induction values obtained by calculation and measuring for different excitation currents:

Curve 1- for rotor,

Curve 2- for stator

On the basis of the presented data it is visible that the deviation is not uniform over the whole measured area, but that deviations are larger at higher induction values. The main cause of deviations is the imperfection of the model not incorporating completely all structure details such as the air gap at the point of the pole pad on the pole wheel, and for bolts for pole stacking. A significant influence is also made by the filling factor for the stator core and the pole that has not taken into consideration by 2D model. The deviation value is also influenced by correctness of measuring that is, within the measured area of 0,1 do $2 \mathrm{~T}$, within the scope of $\pm 2 \%$.

An analysis has been made to explain the form of magnetic induction time dependence using the calculation applying MKE. Another two additional and modified generator modes have been made by MagNet software package. The damping cage was removed from the first model to enable determination of the influence of stator core grooves and the damping cage to the magnetic induction form. The rotor pole shoe height has been changed in the second model to enable determination of the influence of saturation at the rotor pole shoe parts.

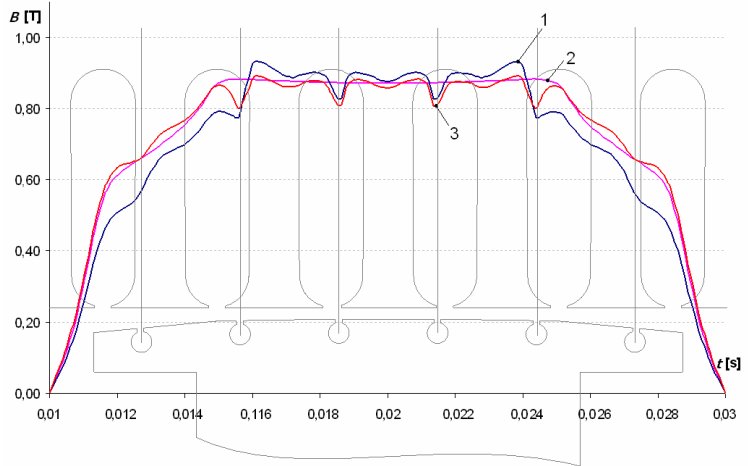

Figure 12. Time dependence of magnetic induction on the top of the stator core tooth achieved by a calculation during idling: Curve 1- with damping cage, Curve 2- without dumping cage, Curve 3- with damping cage, but with the changed rotor pole shoe (as on Figure 13. dotted line). 


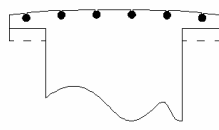

Figure 13. Pole shoe display

Figure 12. shows time dependences of magnetic induction on the top of the stator core tooth corresponding to the point of installation of the Hall probe obtained by real model calculations, and modified models during idling at excitation current of $53 \mathrm{~A}$. Results of field calculation on the model without a damping cage are shown by curve 2 on Figure 12. In addition to a decrease of magnetic induction values at points that correspond to the damping cage two surpasses are visible as well. Results of calculation on the model with the changed pole shoe height (Figure 13.) are represented by curve 3 on Figure 12. From the results it is visible that the surpasses of magnetic induction are decreased as a consequence of a decrease in saturation in parts of the pole shoe.

Time dependences of magnetic induction from the top of the rotor pole shoe are shown on Fig. 14. The wave form show decreases in inductions that occur at moments when a slot aperture comes above the point of measuring. When we count those decreases in one pole pitch, we will obtain the amount corresponding to the number of stator core slots per pole. Damping winding slots decrease the pole shoe surface and the value of magnetic induction is higher for the models having a damping cage.

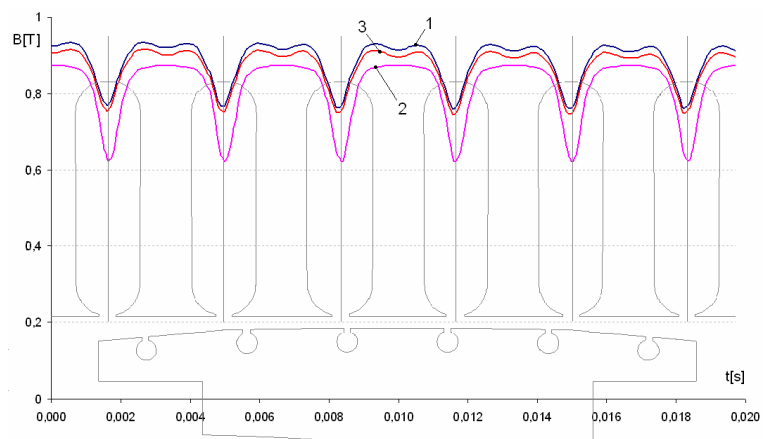

Figure 14. Magnetic induction time dependences on the top of the pole shoe obtained by calculation during idling: Curve 1- with damping cage, Curve 2- without damping cage, Curve 3- with damping cage, but with the changed rotor pole shoe (as on Figure 13 dotted line)

Through a more detailed analyses of magnetic induction time dependence on the pole shoe presented on Figure 13, it can be noticed that the signal surpasses at the moment the Hall probe comes to the slot aperture of the stator and when it leaves it. The stated surpasses are connected with saturation in the stator core tooth caused by slot apertures and the damping cage. Unless they existed the arrangement of magnetic force lines will be as shown on Figure 15. represented by blue dotted lines. Nevertheless, due to stator core slot apertures and the damping cage, the arrangement of magnetic force lines looks like it is presented on Figure 15. represented by red lines.

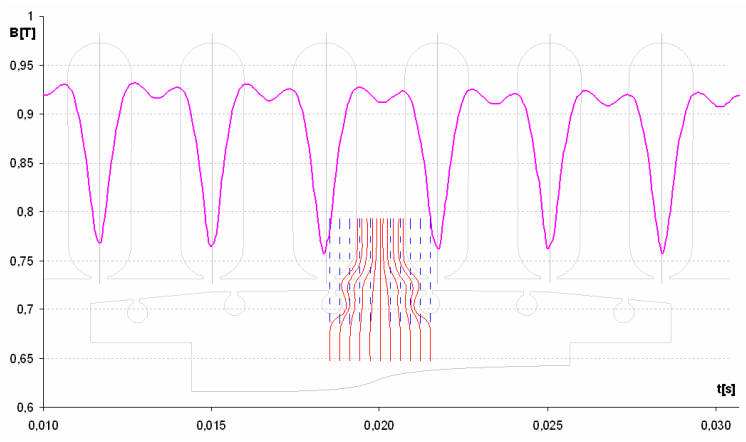

Figure 15. Display of magnetic inductance time dependence on the top of the pole shoe obtained by calculation at excitation current of $53 \mathrm{~A}$

\section{Conclusion}

The comparison of the results of electromagnetic phenomena calculation corresponding to the real geometry of the machines to the results of measuring of the same phenomena is shown. For the needs of calculation, a model was made and software packages applying MKE were used. Measurements were performed through application of the most recent probes and data processing methods enabling precise and reliable calculations. Comparing the measured and the calculated values of magnetic inductions, we have obtained a matching of wave forms, but also deviations, in absolute values, of magnetic inductions in individual points. On the basis of determined deviations, and pursuant the awareness of rotating machine design requirements, validity of the applied calculation for the inspected operation conditions of the generator may be verified. Deviations between the calculated and the measured data have resulted from idling at excitation currents from $0 \%$ to $125 \%$ of the nominal value, and they go up to $6.5 \%$.

Further on, through two iterations of the calculation it is visible that the wave form of induction influences the form of the air gap, as well as of magnetic circuit saturation in parts neighbouring to the air gap.

A certain influence to the increase of calculated values compared to the measured values has been noted due to negligence of structural details.

Performed works indicate the need of further research and study of electromagnetic phenomena in synchronous generators with the aim to verify analytic methods in a wide scope of structural forms.

\section{Bibliography}

[1] Hall probe measurement of magnetic fields, University of Illinois at Urbana-Champaign, Department of Physics 8/1999.

[2] Magnet Version 6.5. "Tutorials", Infolytica Corporation 2000.

[3] Z. Haznadar, Ž. Štih: Elektromgnetizam; Školska knjiga, Zagreb 2000. 